\title{
Dissociating between cardinal and ordinal and between the value and size magnitudes of coins
}

\author{
DANiEl Fitousi \\ Pennsylvania State University, University Park, Pennsylvania
}

\begin{abstract}
In mathematics, the ordinal (relative) magnitude of a numerical object conveys a separate meaning from its cardinal (absolute) magnitude, whereas its physical size bears no inherent relationship to its magnitude. In numerical cognition, the ordinal-cardinal distinction has been scarcely addressed, whereas the size-magnitude distinction has been studied extensively, with the surprising demonstration of an interaction between semantic magnitude and physical size (Besner \& Coltheart, 1979). The present work used coins to study the intricate relations between these meanings. In two experiments, Israeli observers (Experiment 1) and American observers (Experiment 2) performed numerical and physical comparative judgments of coins. Consensual markers of magnitude activation (e.g., the size congruity effect and the distance effect) were obtained. The results of the two experiments converged on the same conclusions. Comparisons of value were governed by ordinal magnitude. Magnitude interfered with comparisons of size, but size did not affect value. The results provided a set of clear dissociations between cardinal and ordinal magnitude and between value and size of coins. They highlight the important role played by ordinal information in magnitude processing.
\end{abstract}

In mathematics, the distinction between ordinal and cardinal magnitudes is fundamental (Frege, 1884; Russell, 1919). The former entails the position occupied by a numerical object in a sequence of elements; the latter denotes the absolute number of elements in a set. In numerical cognition, though, this basic principle has received only limited attention. The aim of the present study was to relate these two senses of magnitude to behavioral regularities with coins. It asked how these highly abstract concepts are used by observers in comparisons of symbolic magnitude and physical size, and whether cardinal and/or ordinal magnitudes exert concrete and distinctive influences on performance with the value and size of coins.

We applied two important markers of magnitude processing to coins. In the well-known distance effect (Moyer, 1973; Moyer \& Landauer, 1967), the time it takes to select the larger member of a pair of numbers or objects decreases as numerical distance separating them increases (e.g., 8 is responded to faster in the $8-2$ pair than in the 8-7 pair). In the size congruity effect (SCE; e.g., Paivio, 1975; Tzelgov, Meyer, \& Henik, 1992), observers are faster to select the physically larger number or object with congruent (2 8) than with incongruent (2 8) pairs. The SCE indicates that semantic magnitude intrudes on the physical judgment, even when it is not required by the task at hand. Both the distance effect and the SCE support the idea that numbers are mapped onto their analog (e.g., cardinal) representation, very much like physical objects (Dehaene, Dupoux, \& Mehler, 1990; Piazza, Pinel, Bihan, \& Dehaene, 2007; Restle, 1970; Shepard, Kilpatrick, \& Cunningham, 1975).

In contrast to the widely held view in numerical cognition - that numerical objects activate their cardinal (absolute) magnitude - it is still possible that both the distance effect and the SCE are generated by the activation of ordinal (relative) magnitude. Moreover, these effects might not be unique to integers. Choplin and Logan (2005) were able to observe the SCE with letter-digit pairs (e.g., c-8). In this study, observers suffered interference from the irrelevant numerical dimension with those letter-digit pairs even though a letter is deprived of numerical magnitude. Choplin and Logan endorsed the argument that ordinal magnitude was activated.

One problem that, perhaps, precluded dissociation between cardinal and ordinal magnitudes with integers (Arabic numerals) might be related to the issue of identifiability. Note that, with Arabic numerals or number words, identical behavioral results are predicted for both cardinal and ordinal magnitudes. Consider the distance effect: An identical form of function relating reduction in response times (RTs) to magnitude is predicted for both cardinal and ordinal distances. A similar problem thwarted the SCE (Fitousi \& Algom, 2006). To obtain a clear dissociation between those two senses, one needs to find a numerical object that allows the following: (1) cardinal and ordi- 
nal magnitudes are disambiguated, (2) physical size and numerical magnitude are present, but are not correlated, and (3) consensual markers of magnitude can be applied. Fortunately, coins meet all of these criteria.

A comment regarding the relation between coins and other canonical numerical formats is in order. Because the distinction between cardinal and ordinal magnitudes is mathematical (Frege, 1884; Russell, 1919), it transcends the format in which magnitudes are presented, be it coins, number words, Arabic numerals, or Roman numerals. Note that coins might or might not be processed like Arabic numerals or other canonical formats. That is an empirical question the present study might be able to answer. The major issue addressed here is whether cardinality and ordinality exert distinctive behavioral signatures on the perception of numerical magnitude and, if so, how. The reason for employing coins is that, unlike other formats, they allow for a clear behavioral dissociation between cardinal and ordinal magnitudes.

A nickel denotes a certain (cardinal) value (e.g., 5 cents), which is completely separate from its ordinal magnitude (e.g., the second coin in the American coinage system). The cardinal monetary value of a coin is ostensibly different from its ordinal magnitude. In addition, in coins, physical size is not confounded with numerical magnitude. This allows one to derive simultaneously various distance effects (i.e., ordinal, cardinal) and the SCE. The form of the distance effect can guide inference on the type of representation (ordinal or cardinal) that governs the perception of coins (see also Dehaene et al., 1990). The SCE (or a lack thereof) can provide further evidence for the activation of magnitude.

Pairing all official Israeli (Experiment 1) and all American (Experiment 2) coins resulted in roughly the same number of congruent and incongruent stimuli. In a congruent pair, the physically larger coin carried higher monetary value than the physically smaller coin, whereas the reverse held true with incongruent pairs. Note that, in the present study, physical size was not manipulated arbitrarily by the experimenter but, rather, reflected real-world situations. Observers performed two tasks. In one block, participants judged the physical size of coins while ignoring numerical value. In another block, participants judged the numerical magnitude while ignoring physical size. If monetary value is activated, irrelevant value should intrude on size judgments and result in an SCE. Conversely, if physical size affects value judgments, a reversed SCE (RSCE) should be observed (Fitousi \& Algom, 2006; Fitousi, Shaki, \& Algom, 2009).

The predictions for the ordinal and cardinal magnitudes are straightforward and, most important, dissociable. If coins are processed with respect to their ordinal magnitude, an ordinal distance effect should be the best predictor of RT. If, on the other hand, coins are processed according to their cardinal magnitude, cardinal distance models should better account for performance. In addition, if coins are processed like Arabic numerals, the pattern of interferences should be asymmetric, with value interfering with size (e.g., SCE), but with size not affecting value.

\section{EXPERIMENT 1}

\section{Method}

Participants. Twenty-four Tel-Aviv University undergraduates participated in fulfillment of course requirements.

Stimuli and Apparatus. All six official Israeli coins were used. Their values, expressed in New Israel Shekels (NIS), were (1) 0.05, (2) 0.1, (3) 0.5 , (4) 1, (5) 5 , and (6) 10 . The coins were round metal disks with diameters of (1) 19.5 , (2) 22 , (3) 26 , (4) 18 , (5) 24 , and (6) $23 \mathrm{~mm}$. Color pictures of the coins' heads were presented in their veridical size and color. All 30 possible pairs were presented. Each member appeared once on the left and once on the right side. Therefore, each pair was presented in two different arrangements in all. Presenting each individual arrangement three times resulted in a block of 90 trials. Order of stimulus presentation was random. The number of congruent and incongruent trials was on par.

The stimuli were generated by an IBM-compatible (PC 486) microcomputer and were displayed on a $600 \times 800$ pixel VGA color monitor. The coins appeared over a white background at the center of the left and right hemifields. They were separated by $15 \mathrm{~cm}$. To avoid adaptation, we introduced a trial-to-trial spatial uncertainty of up to 15 pixels around the target locations. The viewing distance was $60 \mathrm{~cm}$, so that the stimuli appeared at $0.71^{\circ}$ of visual angle to the right and left of fixation.

Procedure. The participants were tested individually in a dimly lit room. Half of the participants started with the physical task, whereas the other half started with the numerical task. Responses were given by pressing a right- or a left-hand key on the computer keyboard, according to the location of the physically (or numerically) larger stimulus. Twenty practice trials preceded the experimental trials in each task (unbeknown to the participants). The stimuli were response terminated. A new pair of stimuli was presented following a 500 -msec interval.

\section{Results}

Size congruity effect. Figure 1 gives the mean RTs for congruent and incongruent conditions for judgments of physical size and monetary value. The respective means were $632 \mathrm{msec}$ for comparisons of physical magnitude and $740 \mathrm{msec}$ for the comparisons of monetary value. The 108-msec advantage in favor of the physical task was reliable $\left[F(1,23)=23.113, M S_{\mathrm{e}}=12,308, p<.0005\right]$. The error rates were $10.5 \%$ for physical comparisons and $7.5 \%$ for the monetary comparisons $[t(23)=1.31, p=$ .204]. The comparisons of numerical value were not affected by irrelevant physical size. Responses on the congruent trials $(M=745 \mathrm{msec})$ were as fast as those on the incongruent trials $(M=735 \mathrm{msec})[t(23)=1.22, p=$ .23]. Participants had lower error rates for the congruent pairs $(8 \%)$ than for the incongruent pairs $(13 \%)$; the difference was not reliable $[t(23)=-1.55, p=.134]$. By contrast, the comparisons of physical size were affected by the irrelevant monetary value. The congruent pairs $(596 \mathrm{msec})$ were compared more quickly than the incongruent pairs (667 msec) $[t(23)=6.59, p<.00005]$, yielding a large SCE of $71 \mathrm{msec}$. This large SCE was mirrored in the accuracy data, with participants making fewer errors in congruent $(1 \%)$ than in incongruent $(14 \%)$ conditions $[t(23)=4.15, p<.005]$. In an overall ANOVA on RTs, a reliable task $\times$ congruity interaction confirmed that this congruity effect was obtained in physical, but not in numerical, comparisons $\left[F(1,23)=38.645, M S_{\mathrm{e}}=\right.$ 999, $p<.00005]$. 


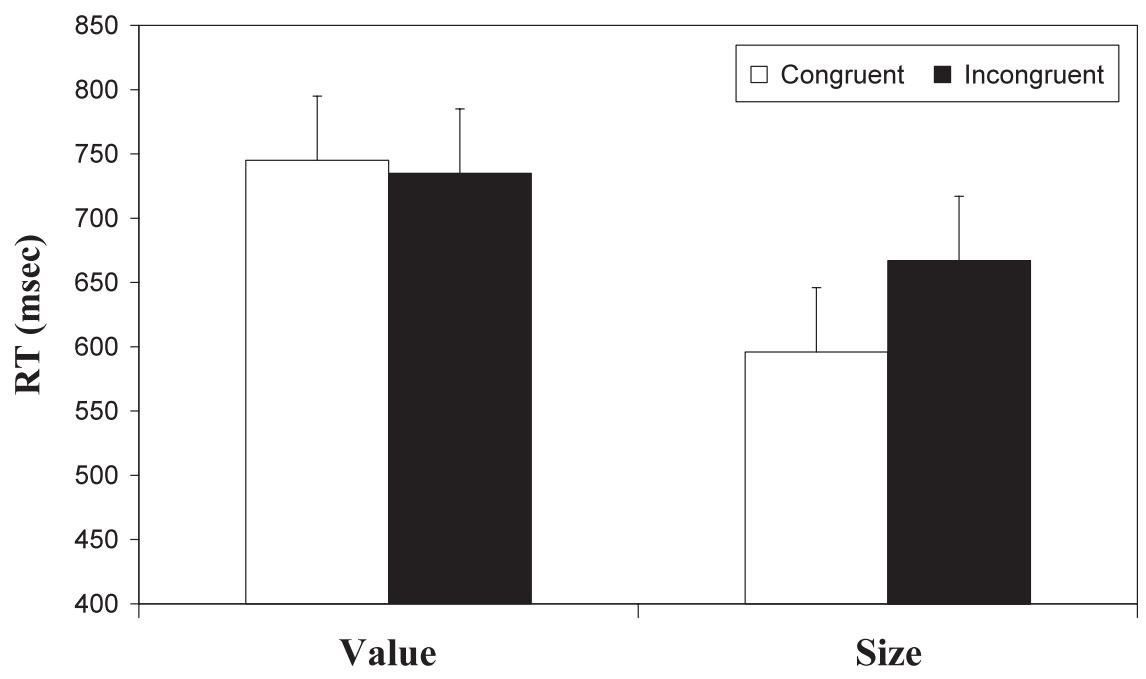

Figure 1. Experiment 1: Size congruity effects with Israeli coins.

Distance effects in the numerical task. In the following analyses, the data were restricted to those coming from the numerical comparative judgment task. The notations "a" and "b" are used to refer to the absolute magnitudes of the first and the second coins presented. First, the influence of the ordinal distance on mean RT was tested. Coins were treated as points equally separated along an ordinal scale (e.g., the distance between the 0.05 NIS coin and the 0.5 NIS coin was 3 unitsequal to the distance between 0.1 NIS and 5 NIS). The ordinal distance for each pair of coins was computed and regressed on mean RT. As can be seen in Figure 2, the model fit was very good $\left[F(1,4)=355.004, M S_{\mathrm{e}}=\right.$ $\left.16.633, p<.000, R^{2}=.992\right]$.

Next, several models of cardinal distance were tested in a similar fashion. The absolute distance $(|a-b|)$ showed the worst fit $\left[F(1,12)=3.57, M S_{\mathrm{e}}=2,603, p=.08\right.$, $\left.R^{2}=.229\right]$. Then, the logarithmic absolute distance $|\log (\mathrm{a} / \mathrm{b})|$ revealed an improved fit $[F(1,6)=5.369, p=$ $\left..068, R^{2}=.518\right]$. Finally, a power function distance was tested by regressing $\log \mathrm{RT}$ on $|\log (\mathrm{a} / \mathrm{b})|[F(1,6)=4.831$, $\left.M S_{\mathrm{e}}=0.002, p=.079, R^{2}=.491\right]$. None of the cardinal models exhibited a reliable effect. The results are quite clear. Ordinal distance accounts for RT better than does cardinal distance.

Distance effects in the physical task. To test whether the perception of physical size of coins is governed by cardinal or ordinal distance effects, we used the diameter of the coins as an independent variable. Data were restricted to those coming from the physical comparison task. Here, "a" and " $b$ " refer to the absolute diameter size of the first and second coins, respectively. First, we tested cardinal

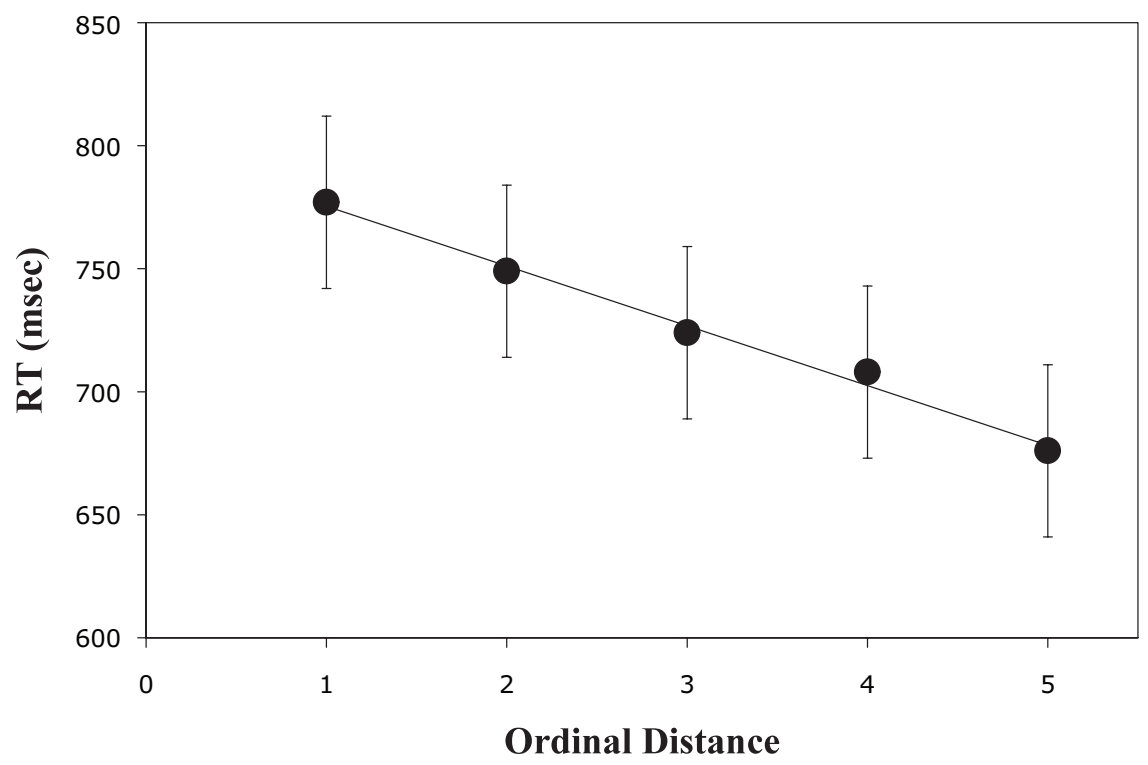

Figure 2. Experiment 1: Ordinal distance effects with Israeli coins. 


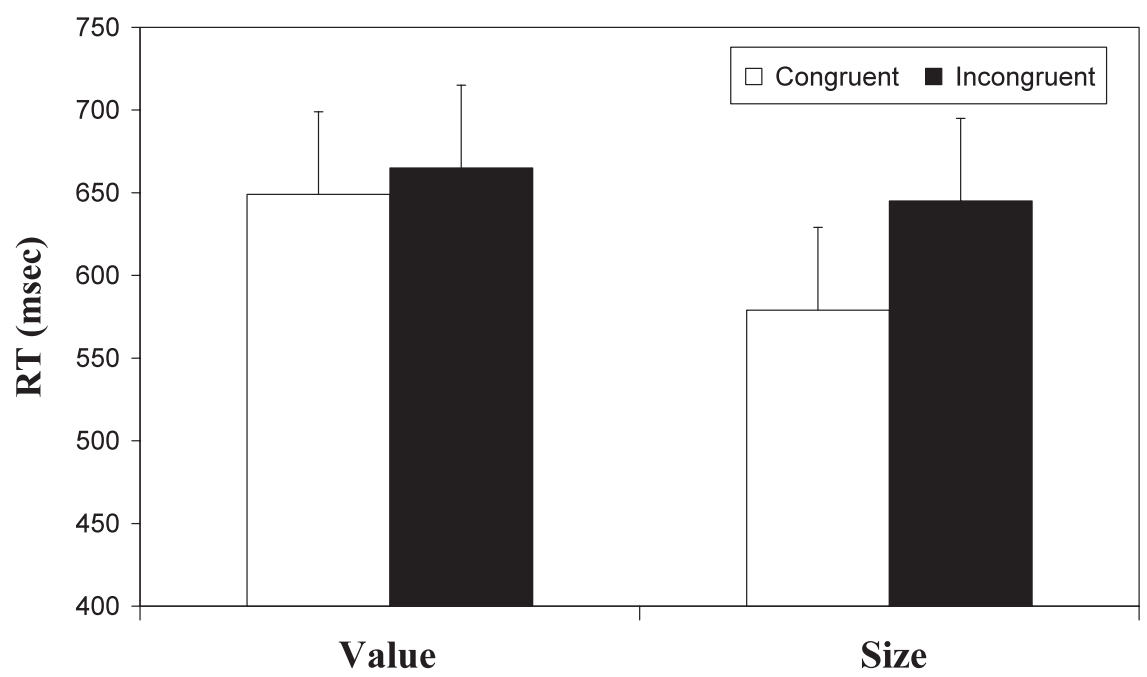

Figure 3. Experiment 2: Size congruity effects with American coins.

models of distance. The $(|\mathrm{a}-\mathrm{b}|)$ model showed the best fit $\left[F(1,10)=66.23, M S_{\mathrm{e}}=43.64, p<.0001, R^{2}=.86\right]$. Next we tested the power function distance, where we regressed $\log \mathrm{RT}$ on $|\log (\mathrm{a} / \mathrm{b})|\left[F(1,10)=57.42, M S_{\mathrm{e}}=\right.$ $\left.0.08, p<.0001, R^{2}=.85\right]$. Then we tested the logarithmic absolute distance $|\log (\mathrm{a} / \mathrm{b})|\left[F(1,10)=66.23, M S_{\mathrm{e}}=\right.$ $\left.0.08, p<.0001, R^{2}=.83\right]$. Finally, we tested an ordinal distance model by assigning numbers from 1 to 6 to each coin according to its diameter. This model outperformed the cardinal models $\left[F(1,5)=28.87, M S_{\mathrm{e}}=0.31, p<\right.$ $\left..05, R^{2}=.90\right]$.

\section{Discussion}

The results of Experiment 1 demonstrate that coins are subjected to both the SCE (Tzelgov et al., 1992) and the distance effect (Moyer \& Landauer, 1967). Mon- etary value interfered with physical comparisons of coins, whereas physical size did not hamper judgments of monetary value. More important, when ordinal and cardinal models of value were fit to RT, ordinal distance was a better predictor of performance than was cardinal distance. Moreover, an ordinal distance effect was found in comparisons of physical size. Therefore, it is highly probable that the SCE observed here reflects the activation of ordinal magnitude. Taken together, these results clearly demonstrate two dissociations: The first is between value and size; the second is between ordinal and cardinal magnitude. According to these outcomes, ordinal magnitude plays a key role in both size and value judgments of coins. These outcomes invite a replication with a different set of stimuli. In the next experiment, American coins were used.

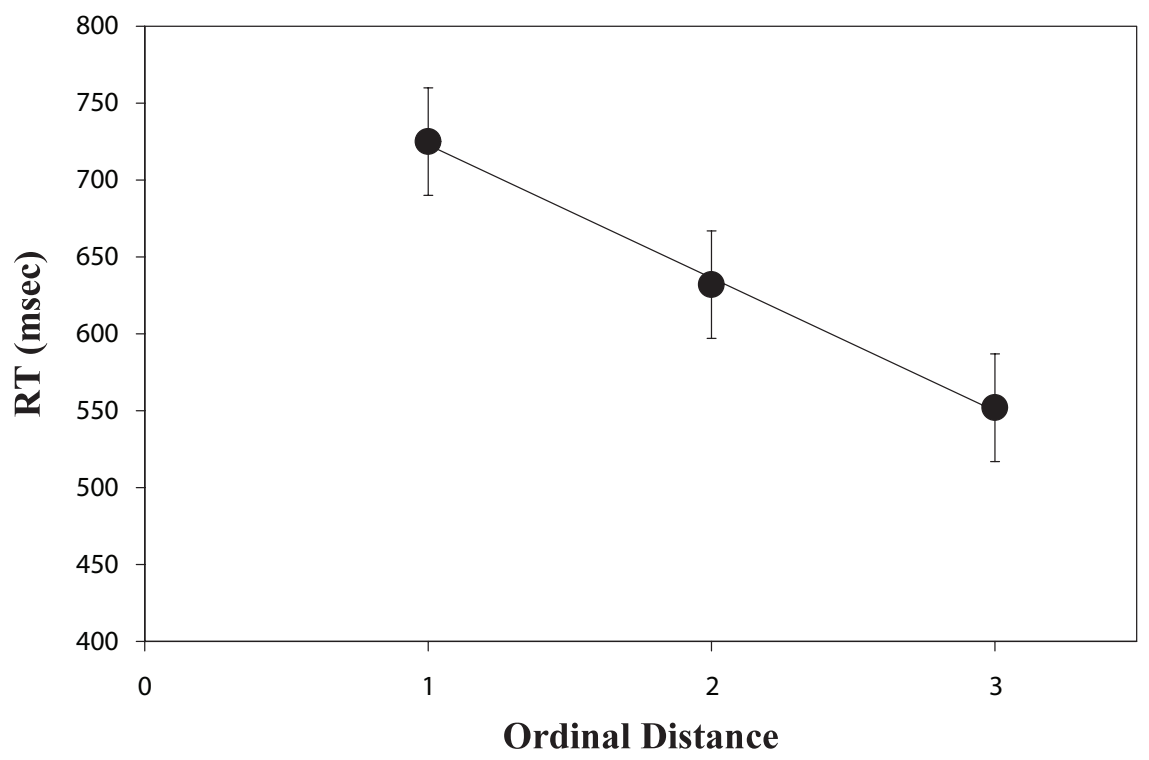

Figure 4. Experiment 2: Ordinal distance effects with American coins. 


\section{EXPERIMENT 2}

\section{Method}

Participants. Eight volunteers from Pennsylvania State University participated.

Stimuli and Apparatus. Four official American coins were used (i.e., a penny, a nickel, a dime, and a quarter). Their values, expressed in U.S. dollars, are (1) 0.01 , (2) 0.05 , (3) 0.1 , and (4) 0.25 . Color pictures of the coins' heads were presented in their veridical size and color. All possible pairs were presented. To equate for the number of congruent and incongruent trials, one member was presented twice. Each member appeared once on the left and once on the right side. Therefore, each pair was presented in two different arrangements in all. Presenting each individual arrangement 5 times resulted in a block of 60 trials. Order of stimulus presentation was random.

A PC-compatible computer with a 19-in. (diagonal) color CRT set to a resolution of $1,024 \times 768$ pixels was used to present all the stimuli and record all the responses. The coins appeared over a white background at the center of the left and right hemifields. They were separated by $15 \mathrm{~cm}$. To avoid adaptation, we introduced a trial-totrial spatial uncertainty of up to 15 pixels around the target locations. The viewing distance was $76 \mathrm{~cm}$.

Procedure. The procedure was similar to that in Experiment 1.

\section{Results}

Size congruity effects. Figure 3 gives the mean RTs for congruent and incongruent conditions for judgments of physical size and monetary value. The mean RTs for the physical and monetary tasks were 612 and $657 \mathrm{msec}$, respectively. The difference was not reliable $[t(7)=$ $1.21, p=.26]$. Error rates amounted to $4.5 \%$ for physical comparisons and $9.2 \%$ for the monetary comparisons $(t<1)$. The comparisons of value were not affected by irrelevant size. Responses on the congruent trials $(M=$ $649 \mathrm{msec}$ ) were as fast as those on the incongruent trials $(M=665 \mathrm{msec})[t(7)=1.38, p=.21]$. Participants had lower error rates for the congruent pairs (4\%) than for the incongruent pairs (14\%); however, the difference was not reliable $(t<1)$. In contrast, the comparisons of physical size were affected by the irrelevant monetary value. The congruent pairs (579 msec) were compared more quickly than the incongruent pairs $(645 \mathrm{msec})[t(7)=4.68, p<$ $.01]$, yielding a large SCE of $66 \mathrm{msec}$. This large SCE was not mirrored in the accuracy data. Participants committed similar rates of errors in the congruent $(4.7 \%)$ and incongruent $(4.3 \%)$ conditions $(t<1)$. In an overall ANOVA on RTs, a reliable task $\times$ congruity interaction confirmed that this congruity effect was obtained in physical, but not in numerical, comparisons $\left[F(1,7)=6.40, M S_{\mathrm{e}}=\right.$ 813.741, $p<.05]$.

Distance effects in the numerical task. The data were restricted to those coming from numerical comparisons. First, ordinal distances were computed for each pair of coins and were regressed on mean RT. As can been seen in Figure 4, a good fit was achieved $[F(1,2)=531.284$, $\left.M S_{\mathrm{e}}=28.167, p<.05, R^{2}=.998\right]$. This replicates the results of Experiment 1. Next, cardinal models of distance were fit to the data. The absolute distance $(|\mathrm{a}-\mathrm{b}|)$ model showed a mediocre fit $\left[F(1,4)=0.93, M S_{\mathrm{e}}=\right.$ 7,581.06, $\left.p=.3, R^{2}=.18\right]$. The logarithmic model $(|\log (\mathrm{a} / \mathrm{b})|)\left[F(1,4)=14.651, M S_{\mathrm{e}}=2,003.3, p<.05\right.$, $\left.R^{2}=.83\right]$ showed a reliable but lower fit to the data. A power function model was tested by regressing log RT on $|\log (\mathrm{a} / \mathrm{b})|$. The fit here was very $\operatorname{good}[F(1,4)=12.407$, $\left.M S_{\mathrm{e}}=0.005, p<.05, R^{2}=.805\right]$, yet not as good as that of the ordinal model. Taken together, the results show that the ordinal model better accounted for RT.

Distance effects in the physical task. The data were restricted to those coming from the physical task. The diameter of the coins was used as an independent variable. Cardinal models of distance were fit to the data. The absolute distance $(|\mathrm{a}-\mathrm{b}|)$ model showed a good fit $[F(1,4)=$ $\left.40.02, M S_{\mathrm{e}}=374.59, p<.01, R^{2}=.90\right]$. The logarithmic model $(|\log (\mathrm{a} / \mathrm{b})|)\left[F(1,4)=12.12, M S_{\mathrm{e}}=1,427.07\right.$, $\left.p<.05, R^{2}=.75\right]$ revealed a reliable but lower fit to the data. The power function model exhibited a good fit as well $\left[F(1,4)=34.37, M S_{\mathrm{e}}=0.001, p<.01, R^{2}=.89\right]$. Next, we fit an ordinal model of distance by first ranking the coins according to their relative physical size (e.g., using the diameter) and then regressing order rank on RT. Although this model accounted for large proportion of the variance, it was not reliable $\left[F(1,2)=23.29, M S_{\mathrm{e}}=\right.$ $\left.260.78, p>.1, R^{2}=.95\right]$. The results clearly demonstrate that, in the case of American coins, cardinal distance is the best predictor of size judgments.

\section{Discussion}

Experiment 2 replicated the results of Experiment 1 with a different set of stimuli. They demonstrate clear dissociations between the size-value and ordinal-cardinal magnitudes of American coins. Monetary value interfered with comparative judgments of coins' sizes, whereas the reverse was not true. This SCE (Fitousi \& Algom, 2006) was found in tandem with an ordinal distance effect in judgments of monetary value (Moyer, 1973; Moyer \& Landauer, 1967). As with Israeli coins, ordinal distance best accounted for comparisons of value. However, unlike Israeli coins, judgments of size were governed by cardinal distance.

\section{GENERAL DISCUSSION}

The present work reveals, for the first time, two important dissociations between physical and semantic properties of coins. These cardinal-ordinal and sizevalue disparities were demonstrated with both Israeli and American coins by using two consensual markers of magnitude processing - the distance effect (Moyer, 1973; Moyer \& Landauer, 1967), and the SCE (Paivio, 1975; Tzelgov et al., 1992). The cardinal-ordinal dissociation is valuable, since it demonstrates how two highly abstract mathematical concepts of magnitude can be disentangled within the same task and without an explicit reference to either of them. The size-value dissociation provides further support for the idea that two types of magnitude can operate differently in both physical and value judgments.

One can argue that coins are processed differently from other numerical notations and, therefore, that the present results are not informative about numerical cognition. We submit that there are compelling reasons to believe that 
coins are processed like integers. First, coins showed an SCE, but not an RSCE, an asymmetry that has been observed with Arabic numerals (Choplin \& Logan, 2005; Tzelgov et al., 1992), but not with number words (Besner \& Coltheart, 1979). Second, researchers have suggested that Arabic numerals are processed like pictures (Damian, 2004; Fias, Reynvoet, \& Brysbaert, 2001). According to this idea, Arabic numerals, unlike number words, have a direct path to their meaning, bypassing phonological translation. In this sense, coins bear functional similarity to Arabic numerals, more so than number words, and thus it is highly likely that their magnitude is retrieved in a similar fashion.

In case one is not willing to accept the proposed similarity between coins and numbers, our results are still valuable. They highlight the role played by ordinal information in magnitude processing. The SCE and the distance effect have been used in other "nonnumerical" contexts. Moyer (1973) found a distance effect in comparative judgments of animal sizes with animal names and ascribed it to an "internal psychophysics." In yet another seminal article, Paivio (1975) demonstrated an SCE with animal pictures. Therefore, these effects are not exclusively numerical, and the ordinal-cardinal and physical-semantic distinctions underlying them are ubiquitous and informative in various domains of psychology (see, e.g., Moyer \& Dumais, 1978). They might suggest that numbers are not "special" but, rather, are subjected to general magnitude-processing mechanisms (Walsh, 2003). Interestingly, the ordinal properties of objects have been studied extensively in various domains of cognitive psychology (Petrusic, 1992; Shoben, Čech, Schwanenflugel, \& Sailor, 1989) but have received only limited attention in numerical cognition.

At the current stage of research, analogical theories of numerical processing (Piazza et al., 2007) cannot readily account for the role played by ordinal magnitude. However, theories that assume relational magnitude (Ben Nathan, Shaki, Salti, \& Algom, 2009; Choplin \& Logan, 2005; Tzelgov et al., 1992) are more commensurate with the present results. Let us conclude by highlighting the need for further theoretical and empirical investigations of the role played by ordinal and cardinal magnitude in human cognition.

\section{AUTHOR NOTE}

This research was performed while the author was at Tel-Aviv University and at Pennsylvania State University. The author thanks Dan Weiss and three anonymous reviewers for their helpful comments on an earlier version of the manuscript. Correspondence concerning this article should be addressed to D. Fitousi, Department of Neuroscience and Behavioral Disorders, Duke-NUS Graduate Medical School, Singapore (e-mail: daniel.fitousi@duke-nus.edu.sg).

\section{REFERENCES}

Ben Nathan, M., Shaki, S., Salti, M., \& Algom, D. (2009). Numbers and space: Associations and dissociations. Psychonomic Bulletin \& Review, 16, 578-582.

Besner, D., \& Coltheart, M. (1979). Ideographic and alphabetic processing in skilled reading of English. Neuropsychologia, 17, 467-472.

Choplin, J. M., \& Logan, G. D. (2005). A memory-based account of automatic numerosity processing. Memory \& Cognition, 33, 17-28.

Damian, M. F. (2004). Asymmetries in the processing of Arabic digits and number words. Memory \& Cognition, 32, 164-171.

Dehaene, S., Dupoux, E., \& Mehler, J. (1990). Is numerical comparison digital? Analogical and symbolic effects in two-digit number comparison. Journal of Experimental Psychology: Human Perception \& Performance, 16, 626-641.

Fias, W., Reynvoet, B., \& Brysbaert, M. (2001). Are Arabic numerals processed as pictures in a Stroop interference task? Psychological Research, 65, 242-249.

FITOusi, D., \& Algom, D. (2006). Size congruity effects with two-digit numbers: Expanding the number line? Memory \& Cognition, 34, 445457.

Fitousi, D., Shaki, S., \& Algom, D. (2009). The role of parity, physical size, and magnitude in numerical cognition: The SNARC effect revisited. Attention, Perception, \& Psychophysics, 71, 143-155.

Frege, G. (1884). Die Grundlagen der Arithmetik. Jena: Hermann Pohle.

Moyer, R. S. (1973). Comparing objects in memory: Evidence suggesting an internal psychophysics. Perception \& Psychophysics, 13, 180-184.

Moyer, R. S., \& Dumais, S. T. (1978). Mental comparison. In G. H. Bower (Ed.), The psychology of learning and motivation (Vol. 12, pp. 117-155). New York: Academic Press.

Moyer, R. S., \& Landauer, T. K. (1967). Time required for judgments of numerical inequality. Nature, 215, 1519-1520.

Paivio, A. (1975). Perceptual comparisons through the mind's eye. Memory \& Cognition, 3, 635-647.

Petrusic, W. M. (1992). Semantic congruity effects and theories of the comparison process. Journal of Experimental Psychology: Human Perception \& Performance, 18, 962-986.

Piazza, M., Pinel, P., Bihan, D. L., \& Dehaene, S. (2007). A magnitude code common to numerosities and number symbols in human intraparietal cortex. Neuron, 53, 293-305.

RestLE, F. (1970). Speed of adding and comparing numbers. Journal of Experimental Psychology, 83, 274-278.

RusSELL, B. (1919). Introduction to mathematical philosophy. London: Allen \& Unwin.

Shepard, R. N., Kilpatrick, D. W., \& Cunningham, J. P. (1975). The internal representation of number. Cognitive Psychology, 1, 82-138.

Shoben, E. J., Čech, C. G., Schwanenflugel, P. J., \& Sailor, K. M. (1989). Serial position effects in comparative judgments. Journal of Experimental Psychology: Human Perception \& Performance, 15, 273-286.

Tzelgov, J., Meyer, J., \& HeniK, A. (1992). Automatic and intentional processing of numerical information. Journal of Experimental Psychology: Learning, Memory, \& Cognition, 18, 166-179.

WaLsh, V. (2003). A theory of magnitude: Common cortical metrics of time, space and quantity. Trends in Cognitive Sciences, 7, 483-488.

(Manuscript received September 14, 2009; revision accepted for publication June 24, 2010.) 